\title{
Meniscal T1rho and T2 measured with 3.0T MRI increases directly after running a marathon
}

\author{
Christoph Stehling • Anthony Luke • Robert Stahl • \\ Thomas Baum • Gabby Joseph • Judong Pan • \\ Thomas M. Link
}

Received: 17 August 2010 /Revised: 7 October 2010 /Accepted: 11 October 2010 /Published online: 30 October 2010

(C) The Author(s) 2010. This article is published with open access at Springerlink.com

\begin{abstract}
Purpose To prospectively evaluate changes in T1rho and T2 relaxation time in the meniscus using $3.0 \mathrm{~T}$ MRI in asymptomatic knees of marathon runners and to compare these findings with those of age-matched healthy subjects. Material and methods Thirteen marathon runners underwent 3.0 T MRI including T1rho and T2 mapping sequences before, 48-72 $\mathrm{h}$ after, and 3 months after competition. Ten controls were examined at baseline and after 3 months. All images were analyzed by two musculoskeletal radiologists identifying and grading cartilage, meniscal, ligamentous. and other knee abnormalities with WORMS scores. Meniscal segmentation was performed to generate T1rho and T2 maps in six compartments.

Results No differences in morphological knee abnormalities were found before and after the marathon. However, all marathon runners showed a significant increase in T1rho
\end{abstract}

C. Stehling $(\varangle) \cdot$ R. Stahl $\cdot$ T. Baum $\cdot$ G. Joseph $\cdot$ J. Pan $・$

T. M. Link

Musculoskeletal and Quantitative Imaging Group (MQIR),

Department of Radiology and Biomedical Imaging,

University of California,

185 Berry Street, Suite 350 ,

San Francisco, CA 94107, USA

e-mail: christoph.stehling@radiology.ucsf.edu

C. Stehling

Department of Clinical Radiology, University of Muenster, Muenster, Germany

A. Luke

Department of Orthopedic Surgery, University of California, San Francisco, CA, USA

R. Stahl

Department of Clinical Radiology,

Ludwig Maximilians University of Munich,

Munich, Germany and $\mathrm{T} 2$ values after competition in all meniscus compartments $(p<0.0001)$, which may indicate changes in the biochemical composition of meniscal tissue. While T2 values decreased after 3 months T1rho values remained at a high level, indicating persisting changes in the meniscal matrix composition after a marathon.

Conclusion T2 values in menisci have the potential to be used as biomarkers for identifying reversible meniscus matrix changes indicating potential tissue damage. T1rho values need further study, but may be a valuable marker for diagnosing early, degenerative changes in the menisci following exercise.

Keywords MRI $3.0 \mathrm{~T} \cdot \mathrm{T} 2$ mapping $\cdot \mathrm{T} 1$ rho mapping . Meniscus · Marathon

\section{Introduction}

Running has been shown to be beneficial for different body systems: it decreases the risk of cardiovascular disease, diabetes mellitus, and mental illness [1,2]. Some studies seem to indicate that moderate levels of running do not increase the risk of osteoarthritis (OA) in the knees for healthy people and that physical activity may have a protective effect [3-5]. Excessive running, acute trauma or chronic injury from running, however, can accelerate the onset of OA [6, 7].

Controversial results from MRI findings and marathon running have been reported previously [8-14]. Several studies indicated that marathon running causes no short-term or medium-term damage. In most studies no immediate injury to knee structures was reported $[8,10,12]$. Kursunoglu-Brahme et al. [11], however, found increased meniscal signal intensity on MRI after jogging. The authors mentioned that the clinical 
significance of these findings is uncertain. Also, Krampla et al. [9] reported minor meniscal signal alterations that were slightly more noticeable immediately after running, but returned to normal 6 weeks after the competition. To better understand running associated alterations and their significance a sensitive technique for detecting early structural and functional changes of the meniscus would be imperative and may have substantial implications in preventing disease progression and providing early treatment.

Morphological defects of meniscus and cartilage visualized with MRI are preceded by early degeneration of their biological matrix; this includes an alteration of proteoglycan (PG) turnover, changes in the collagen composition, and elevation of water content [15-21]. Changes in the matrix are an early and key finding in the evolution of OA. Magnetic resonance imaging (MRI) sequences have been developed to study the cartilage and meniscus matrix non-invasively; these include T2, T1rho (T1 relaxation time in a rotating frame) and dGEMRIC (delayed gadolinium-enhanced MR imaging of cartilage). Using dGEMRIC the PG concentration can be quantified. The MR contrast agent will be more concentrated in tissues with low PG concentrations and thus abnormal cartilage or meniscus matrix composition [15, 21, 22]. T1rho describes the spin-lattice relaxation in the rotating frame, and changes in the extracellular matrix of cartilage like the loss of PG, may be reflected in measurements of T1rho due to less restricted motion of water protons [16, 19, 20, 23]. Rauscher et. al. [16] reported promising results using this technique to quantify degenerative changes in the meniscal matrix. They performed meniscal measurements of T1rho and T2 in healthy subjects and patients with osteoarthritis and demonstrated that meniscal matrix measurements could be used to differentiate healthy subjects from individuals with early OA. T2 relaxation time mapping is sensitive to a wide range of water interactions in tissue and in particular depends on the content, orientation and anisotropy of collagen and may therefore also play a role in assessing meniscal degeneration [16-18].

To better understand the implications of long-distance marathon running for meniscal biochemical composition the purpose of our study was to prospectively evaluate changes in T1rho and $\mathrm{T} 2$ relaxation time in the meniscus using 3.0 T MRI in the asymptomatic knees of marathon runners before, 48-72 $\mathrm{h}$ after, and 3 months after a marathon, and to compare these findings with those of age-matched healthy subjects.

\section{Materials and methods}

Subjects and clinical assessment

The authors had control of the data and information submitted for publication. The study was performed in accordance with the rules and regulations of our University Committee for Human Research and was compliant with the Health Insurance Portability and Accountability Act. Written informed consent was obtained from all subjects.

Thirteen non-professional marathon runners (5 male, 8 female, age $32.3 \pm 5.6$ years, BMI $23.8 \pm 2.5$ ) and 10 physically active controls (4 male, 6 female, age $30.5 \pm$ 5.3 years, BMI 23.2 \pm 2.9 ) were recruited for this study.

Specifically, "beginner" level marathon runners were chosen because their training schedules are cumulatively less intense than higher performing runners and involve lower volume training with fewer miles. Marathon runners were recruited from local marathon running clubs, participating in annual city marathons. The inclusion criteria for marathon runners were to:

1. Have not run a marathon in the past 5 months

2. Have no history of significant knee pain (less than 1 month of knee pain in any given year

3. Have not decreased their training habits because of injury

4. Have no history of surgery on the knee, hip or ankle

5. Have a BMI of greater than 18 or less than 30

The runners underwent a pre-participation screening to ensure no symptoms of knee pain. At the time of acquiring the images before the marathon their running distances were less then 10 miles. After the measurement the long distance training runs were started. All runners underwent a tapering period of 1-2 weeks before the marathon and confirmed that they had no plans to do any marathon training within 3 months after the race. All controls performed recreational sports (such as hiking, bicycling, downhill skiing), but not marathon running, and had no history of knee injury or knee pain as the marathon subjects. The controls were asked not to perform sports for 2 days before the MRI examination to avoid exerciseinduced changes that could affect the MR measurement.

Imaging techniques

The knee joint of the non-dominant leg was imaged on a 3.0 T system (Signa; GE Medical Systems, Waukesha, WI, USA) using a quadrature transmit/receive coil dedicated to knee imaging (Clinical MR Solutions, Brookfield, WI, USA). All marathon runners were examined within the 3 weeks before the race (baseline, BL) and 48-72 $\mathrm{h}$ after the competition (follow-up 1, FU1). In addition, 9 marathon runners were examined 3 months (range: 11-14 weeks) after the marathon (follow-up 2, FU2). Four of the thirteen marathon runners did not attend this examination; 2 had moved in the meantime and 2 refused to undergo the follow-up examination. In the active controls two MR examinations were performed, one at baseline and the follow-up MRI after 3 months. 
The morphology of the cartilage and other knee structures and the integrity of the meniscus were assessed by using:

1. A coronal T1-weighted fast-spin-echo (FSE) sequence with a time to repeat/time to echo $(\mathrm{TR} / \mathrm{TE})=667 /$ $10 \mathrm{~ms}$, acquisition matrix of 384 pixels $\times 256$ pixels, field ov view (FOV) of $13 \mathrm{~cm}$, in-plane spatial resolution of $0.254 \times 0.254 \mathrm{~mm}^{2}$, bandwidth $31.25 \mathrm{kHz}$, acquisition time $6: 32 \mathrm{~min}$ and slice thickness $3 \mathrm{~mm}$

2. A coronal intermediate-weighted fat-suppressed FSE sequence $(\mathrm{TR}=4,300 \mathrm{~ms}, \mathrm{TE}=49 \mathrm{~ms}, \mathrm{ETL}=9)$, matrix $320 \times 256$ pixels, FOV of $13 \mathrm{~cm}$, in-plane spatial resolution $0.254 \times 0.254 \mathrm{~mm}^{2}$, bandwidth $31.25 \mathrm{kHz}$, acquisition time $8: 46 \mathrm{~min}$, and slice thickness $4.3 \mathrm{~mm}$

3. An axial intermediate-weighted fat-suppressed FSE sequence (parameters as for 2.)

4. A sagittal fat-saturated intermediate-weighted FSE sequence (IM-w FSE) with TR/TE 4,300/51 ms, ETL= 9 and acquisition time 12:42 min, FOV $16 \mathrm{~cm}$ (matrix $512 \times 256$ pixels), in-plane resolution $0.293 \times 0.293 \mathrm{~mm}^{2}$, slice thickness $2 \mathrm{~mm}$, and section gap of $0.5 \mathrm{~mm}$

5. T1-weighted three-dimensional (3D) high-spatialresolution volumetric fat-suppressed spoiled gradientecho (SPGR) sequence (TR=20 ms, TE $20 / 7.5 \mathrm{~ms}$ ), flip angle $12^{\circ}$, matrix 512 pixels $\times 512$ pixels, in-plane resolution $0.293 \times 0.293 \mathrm{~mm}^{2}$, FOV of $16 \mathrm{~cm}, 0.75$ excitations, acquisition time 7:37 min, and slice thickness of $1 \mathrm{~mm}$

6. Sagittal T1rho and

7. Sagittal T2 mapping sequences were used to assess the meniscal matrix

Three-dimensional T1rho-weighted images were obtained by using spin-lock techniques and SPGR image acquisition, as previously described in detail by $\mathrm{Li}$ et al. [24]. In short, the sagittal three-dimensional T1rhoweighted imaging sequence consisted of two parts: magnetization preparation for imparting T1rho contrast and an elliptically centered segmented three-dimensional SPGR acquisition immediately after the T1rho preparation during transient signal evolution. The main parameters used for this sequence were as follows: $\mathrm{TR}=9.3 \mathrm{~ms}, \mathrm{TE}=3.7 \mathrm{~ms}$; 14-cm field of view; $256 \times 192$ pixel matrix; $3-\mathrm{mm}$ slice thickness; receiver bandwidth $31.25 \mathrm{kHz} ; 48$ views per segment; recovery time $1.5 \mathrm{~s}$; spin-lock times $0,10,40$, and $80 \mathrm{~ms}$; and spin-lock frequency $500 \mathrm{~Hz}$, acquisition time 12:42 min. Sagittal three-dimensional T2 maps were acquired by adding a non-selective $\mathrm{T} 2$ preparation imaging sequence to the SPGR sequence used for T1rho mapping and using a TR of $2,000 \mathrm{~ms}$ and echo times of 4.1, 14.5, 25.0 , and $45.9 \mathrm{~ms}$. All other prescription parameters for the T2-weighted sequence were identical to those used for the
T1rho sequence, and the total acquisition time was 10:36 min.

Image analysis

\section{Semiquantitative, morphological analyses}

Magnetic resonance images of the right knee were reviewed on picture archiving communication system (PACS) workstations (Agfa, Ridgefield Park, NJ, USA) by two musculoskeletal radiologists separately with 20 and 4 years' experience in musculoskeletal imaging; if scores were not identical consensus readings by both radiologists were performed. During the reading session ambient light was reduced and no time constraints were used. Radiologists had access to all sequences acquired from the subjects.

A whole-organ magnetic resonance imaging score (WORMS) was used to evaluate the images for OArelated abnormalities of the knee [25]. Findings in six regions at the knee were recorded, at the patella, trochlea, medial and lateral femur, and medial and lateral tibia, condensing the original regions described in WORMS from 15 to 6 . Using the semi-quantitative scoring system the following joint structures were separately evaluated: menisci, cartilage, bone marrow edema pattern (BMEP), joint effusion, ligaments, osteophytes, subarticular cysts, flattening or depression of the articular surfaces, and loose bodies.

Cartilage signal and morphology was scored using an eight-point scale: $0=$ normal thickness and signal; $1=$ normal thickness, but increased signal on T2-weighted images; $2.0=$ partial-thickness focal defect, $<1 \mathrm{~cm}$ at its greatest width; $2.5=$ full-thickness focal defect, $<1 \mathrm{~cm}$ at its greatest width; $3=$ multiple areas of partial-thickness (Grade 2.0) defects intermixed with areas of normal thickness, or a Grade 2.0 defect wider than $1 \mathrm{~cm}$, but $<75 \%$ of the region; $4=$ diffuse $(\geq 75 \%$ of the region) partial-thickness loss; $5=$ multiple areas of fullthickness loss (grade 2.5) or a grade 2.5 lesion wider than $1 \mathrm{~cm}$, but $<75 \%$ of the region; $6=$ diffuse ( $\geq 75 \%$ of the region) full-thickness loss.

Alterations in meniscal morphology were assessed separately in six regions (medial and lateral: anterior, body, posterior) using a four-level scale $(0$, normal; 1 , intrasubstance abnormalities; 2 , non-displaced tear; 3 , displaced or complex tear; 4, complete destruction/maceration). Subchondral BMEP was defined as poorly marginated areas of increased signal intensity in the normal subchondral and epiphyseal bone marrow on fat-suppressed T2-weighted FSE images. This feature was graded from 0 to 3 based on the extent of regional involvement: $0=$ none; $1=<25 \%$ of the region; $2=$ $25-50 \%$ of the region; $3=>50 \%$ of the region. Ligaments and joint effusion were evaluated using a four-point scale from 0 to $3(0=$ no lesion, $1=$ Grade 1 sprain, $2=$ Grade 2 sprain, $3=$ Grade 3 sprain for ligaments; $0=$ normal, $1=<33 \%$ of 
maximum potential distention, $2=33-66 \%$ of maximum potential distention, $3=>66 \%$ of maximum potential distention for joint effusion). Based on the MR findings a knee was defined as abnormal if a WORMS value of $\geq 1$ was found in any of the subregions evaluated.

Cartilage lesions were also graded using the MRI classification described by Recht et al. [26] based on the arthroscopic Noyes and Stabler scoring system [27]: grade I lesions were defined as having areas of heterogeneous signal intensity on fat-saturated IW FSE sequences; grade II lesions, as cartilage defects that involved less than half of the articular cartilage thickness; grade III lesions, as cartilage defects involving more than half of the cartilage but less than full thickness; and grade IV lesions, as fullthickness cartilage defects exposing the bone.

\section{Meniscal T1rho and T2 measurements}

Images were transferred to a remote SUN/SPARC workstation (Sun Microsystems, Mountain View, CA, USA). T2 and T1rho maps were created. Meniscus segmentation was performed by using in-house software developed with an Interactive Display Language (IDL; Research Systems, Boulder, CO, USA) environment. An IDL routine was used to simplify the drawing of splines delineating meniscal areas (Fig. 1). Tissue contrast was excellent and also fluid was well shown on the sagittal T2 and T1rho maps, thus allowing meniscal segmentation in these images. In each medial and lateral meniscus, distinct regions were defined and segmentation was performed in the individual $\mathrm{T} 2$ and T1rho maps separately. Six different compartments of the meniscus were segmented (medial anterior, medial body,

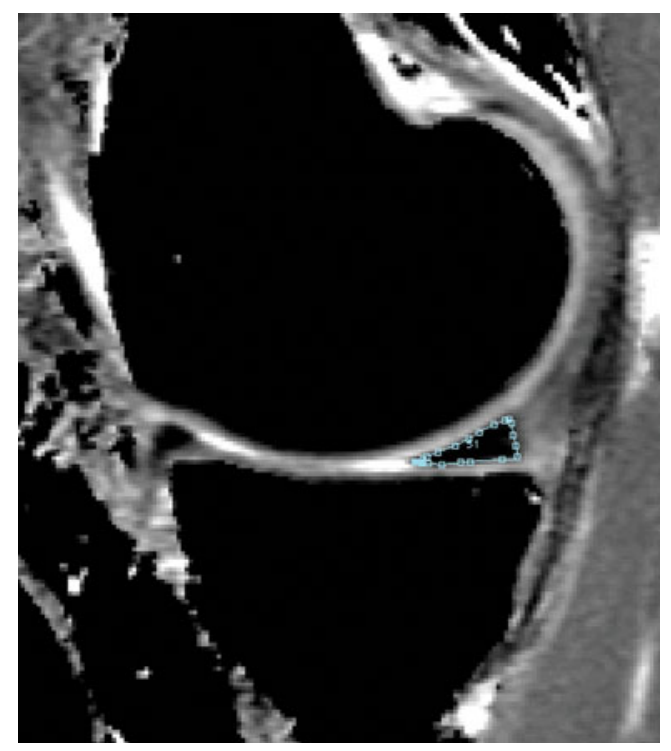

Fig. 1 T1rho map shows the segmented posterior horn of the medial meniscus medial posterior, lateral anterior, lateral body, lateral posterior). The meniscal body was defined mesially as the first section where the anterior and posterior parts of the meniscus were connected and peripherally as the last section showing the meniscus without partial volume effects, which were characterized by a change in the signal intensity of the meniscus compared with the signal intensity of the adjacent sections. The posterior border of the posterior horn of the lateral meniscus was defined by the hiatus popliteus. The fascicles were not included in the analysis. The mesial and peripheral borders separating the meniscus from the roots and capsular ligaments were identified on the basis of partial volume effects and signal intensity changes: only those areas that had the same signal intensity as the more central aspects of the menisci were segmented. After segmentation, an IDL routine was used to calculate the mean $\mathrm{T} 2$ and T1rho values from the regions of interest created on the maps.

\section{Statistics}

All statistical testing was performed with JMP, version 7, software (SAS Institute, Cary, NC, USA). Descriptive statistical evaluation was performed initially. Statistical analysis of differences in age between male and female study subjects and in body mass index were compared by using one-way analysis of variance (ANOVA). Statistical significance for differences in prevalence of knee abnormalities between the groups were determined using Pearson's Chi-squared test. After evaluating the mean T1rho and $\mathrm{T} 2$ values (with standard deviations) for the segmented menisci, the differences according to time points were calculated by using a paired Student's $t$ test.

\section{Reproducibility measurements}

Coefficients of variation (CVs) [28] were calculated to determine the reproducibility of measurements in the entire menisci (medial and lateral menisci combined) and in the meniscal subregions. Five subjects were randomly selected and their menisci were segmented three times by the same investigator. Also, inter-reader reproducibility errors were determined in this way.

\section{Results}

Subject characteristics

There were no significant differences between marathon runners and controls with regard to age $(32.31 \pm 5.6$ years vs $30.45 \pm 5.28$ years; $p<0.4162)$ or BMI $(23.78 \pm 2.53$ vs $23.22 \pm 2.9 ; p<0.6310)$ in the statistical evaluation. 


\section{Focal knee abnormalities}

Table 1 shows the distribution of knee abnormalities in marathon runners and controls. No differences in knee abnormalities were found before and after the marathon. Knee abnormalities did not change over time, either for marathon runners or for volunteers. There were no significant differences in the prevalence of different knee abnormalities in the two groups $(p>0.05)$.

Two of the 13 subjects in the marathon runner group $(15.4 \%)$ and 1 of the 10 subjects in the control group (10\%) had meniscus abnormalities. All meniscus abnormalities were graded as WORMS 1 and were only found in the medial posterior horn. Six of the 13 marathon runners (46.15\%) and 4 of the 10 control subjects ( $40 \%)$ had cartilage abnormalities. Two subjects of each group had abnormalities in more than one region of the cartilage with 8 abnormalities in the marathon runner group and 6 abnormalities in the control group recorded in all six compartments. The cartilage abnormalities were mostly found at the patella ( 6 out of $8,75 \%$ in marathon runners; 4 out of $6,66.67 \%$ in controls) and were graded as mild, either as signal abnormalities or as partial-thickness lesions $<1 \mathrm{~cm}$ (WORMS 1 and 2: 87.5\% in marathon runners, $100 \%$ in controls). BMEP was only found in one subject of each group: joint effusion in one marathon runner. No abnormalities were found in ligaments, also no osteophytes, no subarticular cysts, no depression of the articular surface and no loose bodies could be calculated in either of the groups.

\section{T2 measurements of the menisci}

Figures 2 and 3 and Table 2 demonstrate the results for T2 measurements. In the control group no differences in T2 values (mean: $11.3 \pm 1.2$ vs $11.06 \pm 0.96, p=0.0967$ ) in each compartment were determined between baseline and 3month follow-up. T2 values at baseline did not differ significantly between marathon runners and controls (mean: $11.15 \pm 1.46$ vs $11.3 \pm 1.2 / 11.06 \pm 0.96 ; p=0.5644, p=0.7203$ ).

The marathon runners showed a significant increase in mean T2 values (mean: $11.15 \pm 1.46$ vs $13.36 \pm 1.27, p<$ 0.0001 ) after the competition in all six compartments of both menisci. After 3 months a significant decrease in T2 values (mean: $13.36 \pm 1.27$ vs $11.47 \pm 1.42, p<0.0001$ ) in marathon runners for each compartment was calculated. Marathon runners' T2 values at 3 months did not differ significantly from $\mathrm{T} 2$ values of controls in ANOVA analysis $(11.47 \pm 1.42$ vs $11.3 \pm 1.2 / 11.06 \pm 0.96 ; p=0.106, p=0.519)$.

T1rho measurements of the menisci

Figures 2 and 4 and Table 2 show the results for T1rho. No difference in T1 rho (mean: $15.36 \pm 2.1$ vs $15.38 \pm 2.02$, $p=0.9313$ ) could be demonstrated in the control group between the two timepoints in all six compartments. T1rho values at baseline did not differ significantly between marathon runners and controls (mean: 14.73 \pm 2.11 vs $15.36 \pm 2.1 / 15.38 \pm 2.02 ; p=0.0971, p=0.0826$ ).

The marathon runners demonstrated a significant increase in mean T1rho values (mean: $14.73 \pm 2.11$ vs $17.28 \pm$ $2.45, p<0.0001)$ after competition in all six compartments of both menisci. After 3 months T1rho values remained high (mean: $17.28 \pm 2.45$ vs $17.36 \pm 2.31, p=0.277$ ) in all six compartments and were significantly higher than at baseline $(p<0.05)$. Also, T1 rho values at 3 months were significantly above T1rho values of controls in an ANOVA analysis $(17.36 \pm 2.31$ vs $15.36 \pm 2.1 / 15.38 \pm 2.02 ; p<0.0001$, $p<0.0001)$.

\section{Reproducibility}

Averaged over six compartments, similar errors were found for the two segmentations concerning T1rho $(0.96 \%)$ and $\mathrm{T} 2$ values $(0.97 \%)$. Inter-reader reproducibility errors amounted to $1.2 \%$ for T1rho and $1.47 \%$ for T2 measurements.

\section{Discussion}

The results of this study show that marathon runners have significantly increased $\mathrm{T} 2$ and T1rho values directly after the competition and that $\mathrm{T} 2$ values decreased back to baseline after 3 months, while T1rho values remained at a high level. These findings suggest that using T1rho and T2 measurements, biochemical meniscal matrix changes occur after a marathon that may reflect meniscal injury and may not be completely reversible.

Our results agree with previous studies that marathon running does not induce focal knee MR abnormalities. Also, no significant differences in the prevalence of knee abnormalities in the marathon runner group and control group were found. In an early MR study Shellock et al. also reported that the prevalence of meniscal tears in marathon runners is not higher than the prevalence reported for sedentary persons, and that runners have the same amount of meniscal degeneration as non-runner athletes [12]. While some studies described mild changes in knee joint structures after marathon running, no severe changes were reported: Hohmann et al. examined marathon runners before and after the marathon using MRI and recorded no focal damage at the knee joint due to long distance running [10]. Kursunoglu-Brahme et al. [11] reported an increased prevalence of joint effusion that developed in 5 of the 10 subjects after long distance running. In addition, 5 of the 10 subjects had mildly increased signal intensity within their menisci after jogging. These investigators suggested that 
Table 1 Prevalence, severity, and location of knee abnormalities in the marathon runners and the control group. A whole-organ magnetic resonance imaging score (WORMS) was used to semiquantitatively evaluate the images. Abnormalities were recorded using a WORMS- based threshold of 1 including Grade 1 abnormalities. The knee abnormalities did not change at the different time points, either for marathon runners or for controls

\begin{tabular}{|c|c|c|c|c|c|}
\hline & \multicolumn{2}{|c|}{ Marathon runners } & \multicolumn{2}{|l|}{ Controls } & \multirow{2}{*}{$\begin{array}{l}\text { Statistical analysis }(p) \\
\text { Chi-squared (Pearson's) }\end{array}$} \\
\hline & Number & Percentage & Number & Percentage & \\
\hline Meniscus lesions in subjects & $2 / 13$ & 15.4 & $1 / 10$ & 10 & 0.7039 \\
\hline Meniscus lesions total & 2 & & 1 & & \\
\hline Medial anterior & 0 & 0 & 0 & 0 & \\
\hline Medial body & 0 & 0 & 0 & 0 & \\
\hline Medial posterior & $2 / 2$ & 100 & $1 / 1$ & 100 & \\
\hline Lateral anterior & 0 & 0 & 0 & 0 & \\
\hline Lateral body & 0 & 0 & 0 & 0 & \\
\hline Lateral posterior & 0 & 0 & 0 & 0 & \\
\hline WORMS 1 & $2 / 2$ & 100 & $1 / 1$ & 100 & \\
\hline WORMS 2 & 0 & 0 & 0 & 0 & \\
\hline WORMS 3 & 0 & 0 & 0 & 0 & \\
\hline WORMS 4 & 0 & 0 & 0 & 0 & \\
\hline Cartilage lesions in subjects & $6 / 13$ & 4615 & $4 / 10$ & 40 & 0.7679 \\
\hline Cartilage lesions total & 8 & & 6 & & \\
\hline Patella & $6 / 8$ & 75 & $4 / 6$ & 66.67 & \\
\hline Trochlea & 0 & 0 & $2 / 6$ & 33.34 & \\
\hline MFC & $1 / 8$ & 12.5 & 0 & 0 & \\
\hline LFC & 0 & 0 & 0 & 0 & \\
\hline MT & 0 & 0 & 0 & 0 & \\
\hline LT & $1 / 8$ & 12.5 & 0 & 0 & \\
\hline WORMS 1 & $1 / 8$ & 12.5 & $5 / 6$ & 83.34 & \\
\hline WORMS 2 & $6 / 8$ & 75 & $1 / 6$ & 16.67 & \\
\hline WORMS 2.5 & 0 & 0 & 0 & 0 & \\
\hline WORMS 3 & $1 / 8$ & 12.5 & 0 & 0 & \\
\hline WORMS 4 & 0 & 0 & 0 & 0 & \\
\hline WORMS 5 & 0 & 0 & 0 & 0 & \\
\hline WORMS 6 & 0 & 0 & 0 & 0 & \\
\hline Recht 1 & $1 / 8$ & 12.5 & $5 / 6$ & 83.34 & \\
\hline Recht 2 & $5 / 8$ & 62.5 & $1 / 6$ & 16.67 & \\
\hline Recht 3 & $2 / 8$ & 25.0 & 0 & 0 & \\
\hline Recht 4 & 0 & 0 & 0 & 0 & \\
\hline Bone marrow edema in subjects & $1 / 13$ & 7.7 & $1 / 10$ & 10 & 0.8462 \\
\hline Bone marrow edema total & 2 & & 1 & & \\
\hline Patella & $1 / 2$ & 50 & 0 & 0 & \\
\hline Trochlea & 0 & 0 & $1 / 1$ & 100 & \\
\hline MFC & 0 & 0 & 0 & 0 & \\
\hline LFC & 0 & 0 & 0 & 0 & \\
\hline MT & 0 & 0 & 0 & 0 & \\
\hline LT & $1 / 2$ & 50 & 0 & 0 & \\
\hline WORMS 1 & 0 & 0 & $1 / 1$ & 100 & \\
\hline WORMS 2 & $2 / 2$ & 100 & 0 & 0 & \\
\hline WORMS 3 & 0 & 0 & 0 & 0 & \\
\hline Joint effusion in subjects & $1 / 13$ & 7.7 & 0 & 0 & 0.3698 \\
\hline Joint effusion total & 1 & & 0 & & \\
\hline WORMS 1 & $1 / 1$ & 100 & 0 & 0 & \\
\hline WORMS 2 & 0 & 0 & 0 & 0 & \\
\hline WORMS 3 & 0 & 0 & 0 & 0 & \\
\hline
\end{tabular}



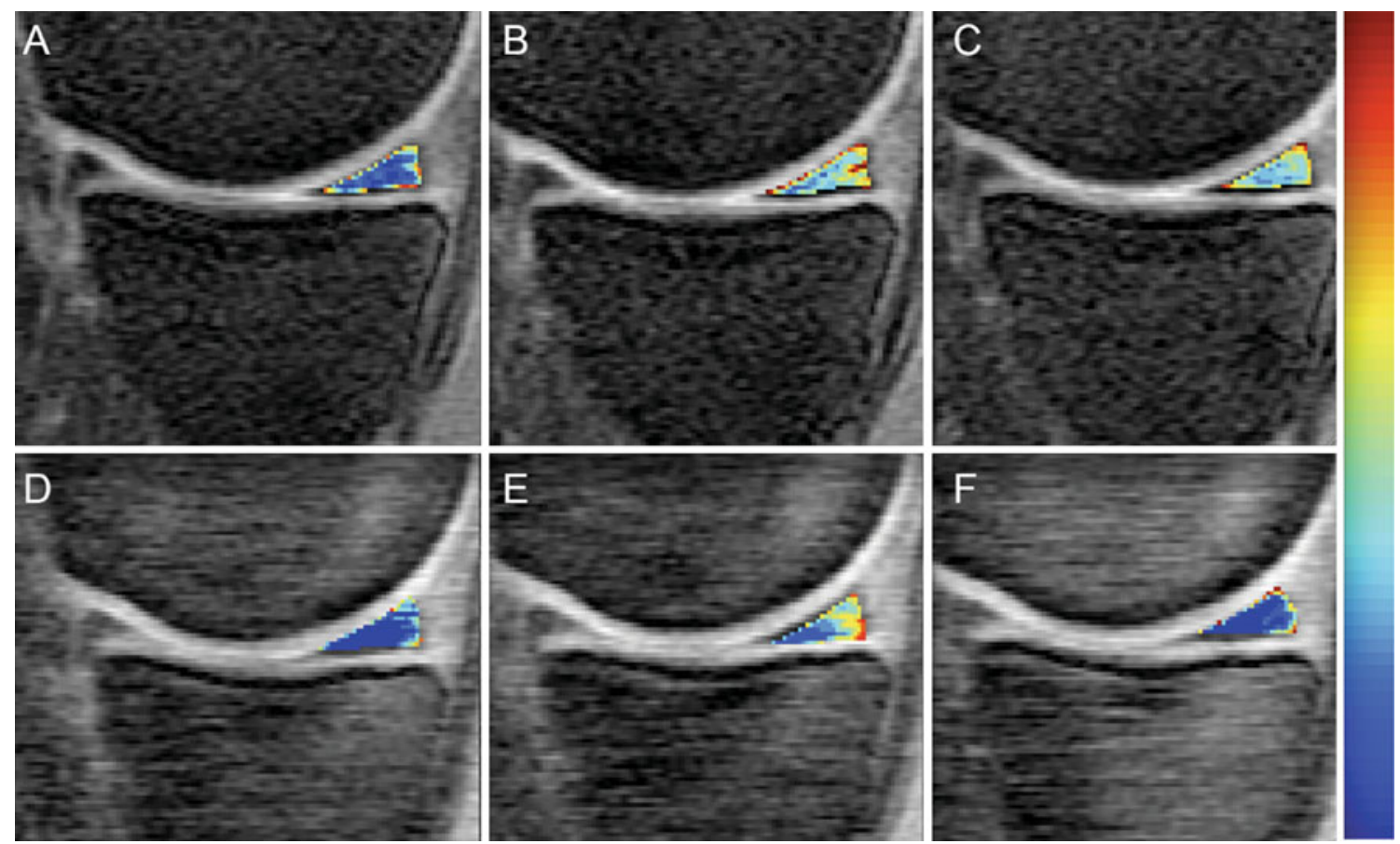

Fig. 2 Representative MR images show the posterior horn of the medial meniscus overlaid with a-c T1rho and $\mathbf{d}-\mathbf{f}$ T2 color maps at three different time points. Images a and $\mathbf{d}$ show color maps at baseline. The change in the colors indicates differences in the meniscal matrix and an increase in the water content after the marathon (b,e). Three months after the marathon persistent changes for T1rho are clearly displayed (c). T2 values decrease back to baseline after 3 months (f) jogging frequently leads to acute changes in the knee, which could be demonstrated with MR imaging. The significance of these changes was unknown at that time. Schueller-Weidekamm et al. [14] also reported joint effusions and increased intrameniscal signal after marathon running. However, they concluded that marathon running does not cause severe, acute lesions of cartilage, ligaments, or bone marrow of the knee in well-trained runners. Similar to these results, Krampla et al. [9] performed an MRI study of the knee in marathon runners before and after competition, and reported that minor meniscal signal alterations were slightly more noticeable soon after running, but had returned to normal 6 weeks after the competition. Without exception the increase in pre-existing low-grade signal alterations were transient. These results correspond to our results where $\mathrm{T} 2$ values decreased after 3 months as a sign of decreased water content and swelling.

To the best of our knowledge, however, no study has been performed to date that has obtained quantitative $\mathrm{T} 2$ or T1rho measurements of the meniscus in marathon runners. Mosher et al. [29] examined knee cartilage T2 values of 7 subjects before and immediately after $30 \mathrm{~min}$ of running. There was a statistically significant decrease in $\mathrm{T} 2$ of the superficial $40 \%$ of weight-bearing femoral cartilage after exercise. These investigators assumed that cartilage compression might result in greater anisotropy of superficial collagen fibers. In contrast, we found an increase in T2 values at the meniscus after running, but the examinations after competition in our study were carried after $48-72 \mathrm{~h}$.

T2 relaxation time mapping is currently most frequently used to study the biochemical composition of cartilage: it is sensitive to a wide range of water interactions in tissue and in particular depends on the content, orientation, and anisotropy of collagen [30,31]. The correlation between $\mathrm{T} 2$ and PG is not yet clear. Some studies showed that the depletion of PG had little influence on T2 [32-34]; WatrinPinzano et al., however, found significantly increased T2 with hyaluronidase-induced PG degeneration [35]. Compared with cartilage, meniscus has higher type 1 collagen concentration, which explains the lower T2 values in meniscus compared with hyaline cartilage $\mathrm{T} 2$ values $[16$, 30].

T1rho describes the spin-lattice relaxation in the rotating frame, and changes in the extracellular matrix of cartilage, like the loss of PG, may be reflected in the measurements of T1rho because of the less restricted motion of water protons $[16,17]$. T1rho correlates highly with the $\mathrm{PG}$ content of the cartilage [36]. The concentration of PG in hyaline cartilage is much higher than the $\mathrm{PG}$ amount in the meniscus [37]; also, the factors that contribute to T1rho changes in the meniscus are not clear and need further investigation. In cartilage, changes in collagen and hydra- 

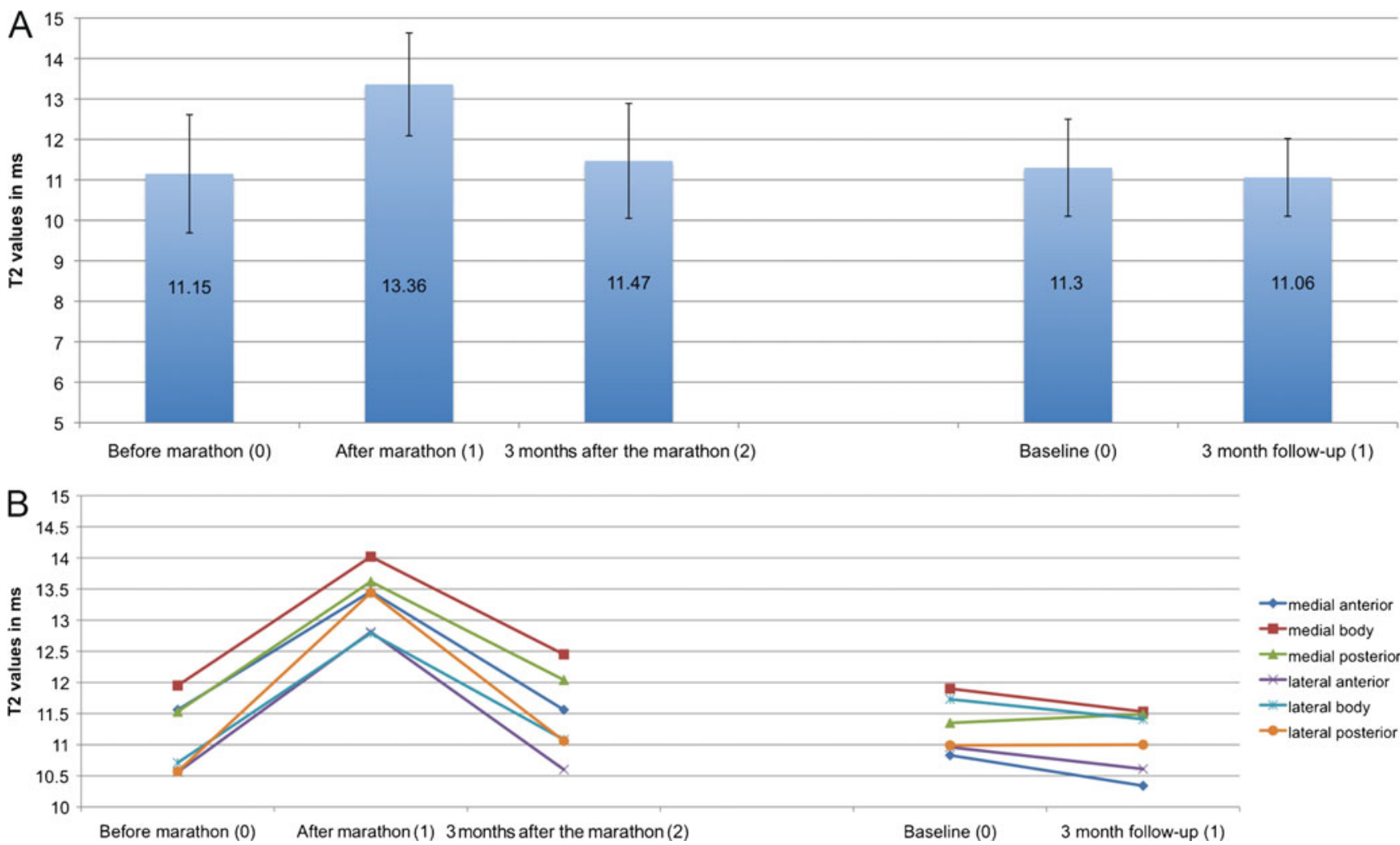

Fig. 3 Diagram of T2 values for $\mathbf{a}$ the whole meniscus and $\mathbf{b}$ for each compartment separately for marathon runners and controls at different time points. All marathon runners showed a significant increase in T2 $(11.15 \pm 1.46$ vs $13.36 \pm 1.27, p<0.0001)$ values $48-72 \mathrm{~h}$ after competition in all six compartments of both menisci. After 3 months a significant decrease in T2 values $(13.36 \pm 1.27$ vs $11.47 \pm 1.42, p<$ 0.0001 ) in marathon runners was calculated. In the controls no difference in T2 values $(11.3 \pm 1.2$ vs $11.06 \pm 0.96, p=0.0967)$ were determined between baseline and the 3-month follow-up

Table $2 \mathrm{~T} 2$ and T1rho values of marathon runners and controls at different time points. Standard deviations (SD) and $p$ values are included. $P$ values marked with an asterisk were statistically significant in a paired $t$ test

\begin{tabular}{|c|c|c|c|c|c|c|c|c|c|c|c|c|c|c|}
\hline \multirow[t]{3}{*}{ Part of the meniscus } & \multicolumn{9}{|c|}{ Marathon runners } & \multicolumn{5}{|c|}{ Controls } \\
\hline & \multicolumn{2}{|c|}{$\begin{array}{l}\text { Before } \\
\text { marathon }(0)\end{array}$} & \multicolumn{2}{|c|}{$\begin{array}{l}\text { After } \\
\text { marathon (1) }\end{array}$} & \multicolumn{2}{|c|}{$\begin{array}{l}3 \text { months after } \\
\text { marathon (2) }\end{array}$} & \multicolumn{3}{|c|}{ Paired $t$ test } & \multicolumn{2}{|c|}{$\begin{array}{l}\text { Baseline } \\
\text { (1) }\end{array}$} & \multicolumn{2}{|c|}{$\begin{array}{l}3 \text { month } \\
\text { follow-up (2) }\end{array}$} & \multirow{2}{*}{$\begin{array}{l}\text { Paired } t \text { test } \\
p\end{array}$} \\
\hline & Mean & $\mathrm{SD}$ & Mean & SD & Mean & SD & $p 0$ vs 1 & $p 0$ vs 2 & $p 1$ vs 2 & Mean & $\mathrm{SD}$ & Mean & SD & \\
\hline \multicolumn{15}{|l|}{$\mathrm{T} 2$} \\
\hline Medial anterior & 11.56 & 1.16 & 13.46 & 0.83 & 11.56 & 0.78 & $0.0001^{*}$ & 0.5912 & $0.0001 *$ & 10.83 & 2.08 & 10.34 & 0.92 & 0.4688 \\
\hline Medial body & 11.95 & 0.95 & 14.02 & 0.84 & 12.45 & 1.07 & $0.0001 *$ & 0.418 & $0.005^{*}$ & 11.9 & 1.01 & 11.53 & 0.6 & 0.1866 \\
\hline Medial posterior & 11.53 & 1.16 & 13.62 & 1.29 & 12.04 & 1.41 & $0.0001 *$ & 0.0536 & $0.0032 *$ & 11.35 & 0.45 & 11.49 & 0.87 & 0.52 \\
\hline Lateral anterior & 10.56 & 1.89 & 12.81 & 1.17 & 10.6 & 1.96 & $0.0001^{*}$ & 0.6753 & $0.0015^{*}$ & 10.96 & 1.24 & 10.61 & 1.14 & 0.2707 \\
\hline Lateral body & 10.71 & 1.39 & 12.79 & 1.21 & 11.08 & 0.83 & $0.0001 *$ & 0.5814 & $0.0001 *$ & 11.73 & 1.07 & 11.41 & 0.86 & 0.1163 \\
\hline Lateral posterior & 10.57 & 1.6 & 13.44 & 1.82 & 11.06 & 1.59 & $0.0001^{*}$ & 0.1404 & $0.0015^{*}$ & 10.99 & 0.55 & 11 & 0.88 & 0.9826 \\
\hline \multicolumn{15}{|l|}{ T1rho } \\
\hline Medial anterior & 15.05 & 1.27 & 17.74 & 1.77 & 17.84 & 1.37 & $0.0001 *$ & $0.0006^{*}$ & 0.4531 & 16.3 & 1.96 & 16.06 & 1.49 & 0.6696 \\
\hline Medial body & 15.05 & 2.62 & 18.11 & 2.65 & 17.71 & 2.36 & $0.0001 *$ & $0.0040^{*}$ & 0.2178 & 16.44 & 2.15 & 16.17 & 1.95 & 0.6163 \\
\hline Medial posterior & 13.65 & 2.85 & 15.81 & 3.34 & 15.88 & 3.26 & $0.0001^{*}$ & $0.0164 *$ & 0.3838 & 14.51 & 2.68 & 15.27 & 2.83 & 0.1609 \\
\hline Lateral anterior & 14.83 & 1.65 & 16.72 & 1.67 & 17.41 & 1.9 & $0.0001 *$ & $0.0032 *$ & 0.9333 & 15.33 & 1.18 & 16.02 & 1.9 & 0.2077 \\
\hline Lateral body & 14.84 & 2.3 & 17.63 & 2.49 & 17.68 & 2.74 & $0.0002 *$ & $0.0021 *$ & 0.1577 & 15.76 & 1.46 & 15.26 & 1.46 & 0.4461 \\
\hline Lateral posterior & 14.97 & 1.64 & 17.67 & 2.16 & 17.65 & 1.94 & $0.0001 *$ & $0.0029^{*}$ & 0.4859 & 13.8 & 1.97 & 13.48 & 1.24 & 0.6382 \\
\hline
\end{tabular}



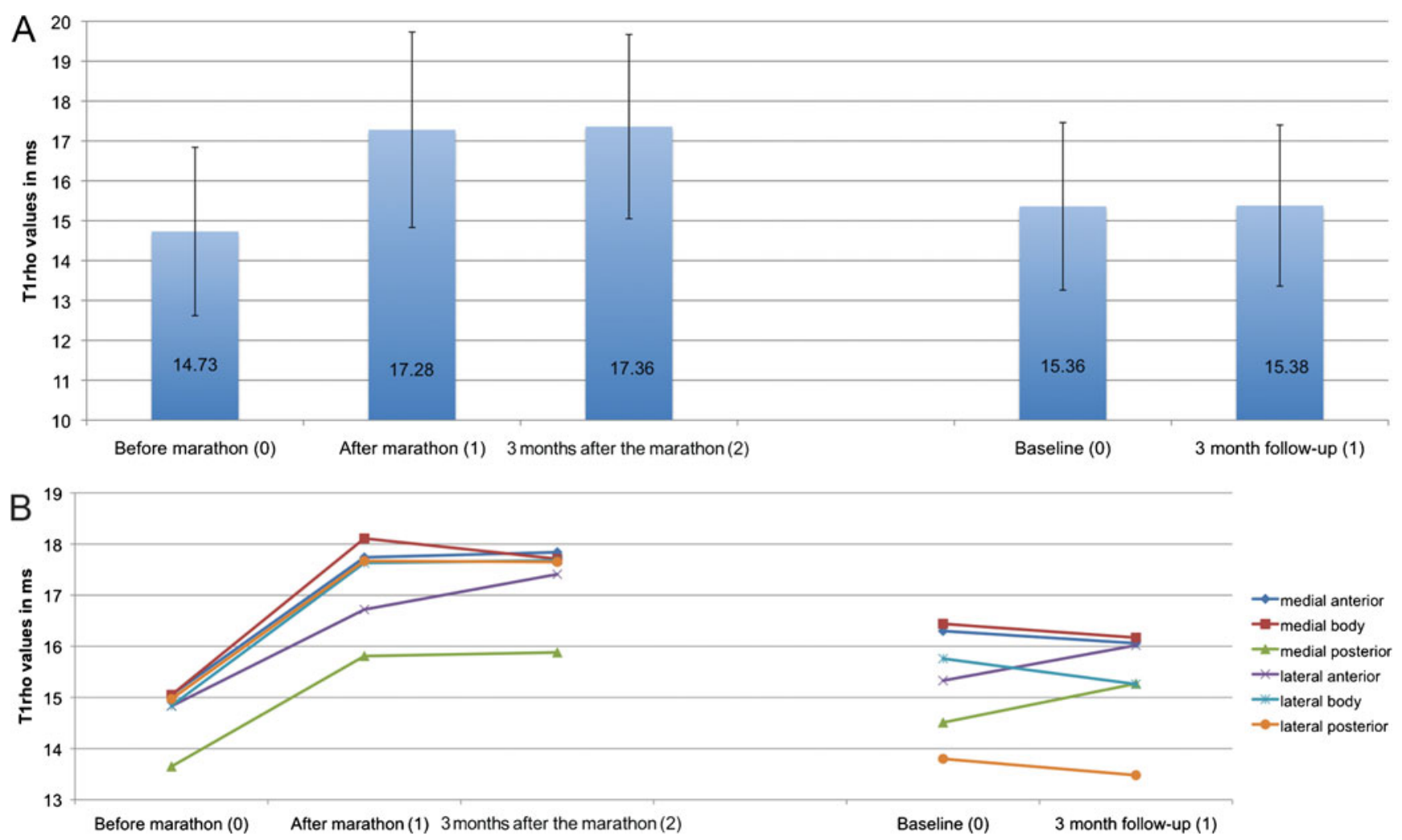

Fig. 4 Diagram of T1rho values for $\mathbf{a}$ the whole meniscus and $\mathbf{b}$ for each compartment for marathon runners and controls at different time points. All marathon runners showed a significant increase in T1rho values $(14.73 \pm 2.11$ vs $17.28 \pm 2.45, p<0.0001)$ after competition in all

tion may affect T1rho. However, even with the current spin-lock frequency $(500 \mathrm{~Hz})$ of clinical MR imagers, T1rho values are approximately $30 \%$ higher than $\mathrm{T} 2$ values in cartilage and approximately $20 \%$ higher in the menisci [16]. Spin-locking reduces dipolar interaction, which dominates T2 relaxation. Previous studies showed that chemical exchanges between bulk water and the hydroxyl and amine groups of PG may be important relaxation mechanisms for T1rho in articular cartilage [38]. Studies have also explained that T1rho is more sensitive to PG changes than is $\mathrm{T} 2$ in degenerated cartilage [20,39]. Given that after 3 months T1rho values remained at a high level in our study, one might conclude that running a marathon might induce persisting changes in the meniscal matrix.

Rauscher et al. analyzed meniscal T1rho and T2 values in healthy subjects and patients with osteoarthritis [16]. The results of this study demonstrated that meniscal matrix measurements may be used to differentiate healthy subjects from individuals with early OA. Matrix measurements increased consistently with higher OA grade. In addition, significant correlations between matrix measurements in the meniscus and clinical scores (WORMS) were found. Krishnan et al. [21] analyzed the T1 values of gadoliniumbased contrast material in the meniscus and the relationship six compartments of both menisci. After 3 months T1rho values remained high $(17.28 \pm 2.45$ vs $17.36 \pm 2.31, p=0.277)$. In the controls no difference in T1rho values $(15.36 \pm 2.1$ vs $15.38 \pm 2.02, p=0.9313)$ was determined between baseline and the 3-month follow-up

between this parameter and the $\mathrm{T} 1$ of delayed gadoliniumbased contrast material in the articular cartilage. Their study results showed that the $\mathrm{T} 1$ in the meniscus correlated significantly with that in the articular cartilage (delayed gadolinium-enhanced magnetic resonance imaging of cartilage (dGEMRIC)), potentially demonstrating associated degenerative processes in the knee joint. Using the dGEMRIC technique the PG concentration can be quantified [22]. In an experimental study Pap et al. [40] found similar results. They examined the influence of an excessive running load on the development of knee OA in male Wistar rats. Histological assessment of the knee joint sections revealed a significant increase in osteoarthritic changes with a higher running load.

Limitations of our study have to be acknowledged. The number of subjects recruited to our study is relatively low; only 13 marathon runners could be examined before and after marathon running and only 9 marathon runners completed the follow-up MRI examination after 3 months. As a consequence of this, our study was not able to investigate the influence of marathon running in general on meniscal structure. Although the number of study subjects was low, statistical significance was achieved as the results were consistent and nearly every study subject showed the 
same longitudinal T2 and T1rho changes in each compartment. To better understand whether a persisting increase in T1rho values 3 months after the marathon might be a sign of irreversible changes in the matrix decomposition longterm follow-up examination after 1 or 2 years would be required to monitor the T1rho values. Also, a histological correlation of the $\mathrm{T} 2$ and $\mathrm{T} 1$ rho values would be interesting, but obviously not possible in a clinical study. T1rho may represent a change in the meniscus matrix, but it is not clear what the factors are that contribute to T1rho changes in the meniscus and needs further investigation.

\section{Conclusion}

Our study demonstrates for the first time a relationship between marathon running and changes in the meniscal matrix composition measured non-invasively with $\mathrm{T} 2$ and T1rho MRI techniques. The findings suggest that $48-72 \mathrm{~h}$ after the competition marathon runners have a significantly higher water content shown in T2 values and possible depletion of proteoglycans as suggested by the T1rho value increase. While T2 values decreased after 3 months T1rho values remained at a high level indicating more persistent changes in the meniscal matrix composition after a marathon. Based on our findings we hypothesize that $\mathrm{T} 2$ values in the menisci have the potential to be used as biomarkers for identifying reversible meniscus matrix changes indicating potential tissue damage. T1rho values need further study, but may be a valuable marker for diagnosing early degenerative changes in the menisci following exercise.

Open Access This article is distributed under the terms of the Creative Commons Attribution Noncommercial License which permits any noncommercial use, distribution, and reproduction in any medium, provided the original author(s) and source are credited.

\section{References}

1. Thompson PD, Franklin BA, Balady GJ, et al. Exercise and acute cardiovascular events placing the risks into perspective: a scientific statement from the American Heart Association Council on Nutrition, Physical activity, and Metabolism and the Council on Clinical Cardiology. Circulation. 2007;115 (17):2358-68.

2. Haskell WL, Lee IM, Pate RR, et al. Physical activity and public health: updated recommendation for adults from the American College of Sports Medicine and the American Heart Association. Med Sci Sports Exerc. 2007;39(8):1423-34.

3. Lane NE, Oehlert JW, Bloch DA, et al. The relationship of running to osteoarthritis of the knee and hip and bone mineral density of the lumbar spine: a 9 year longitudinal study. J Rheumatol. 1998;25(2):334-41.
4. Sohn RS, Micheli LJ. The effect of running on the pathogenesis of osteoarthritis of the hips and knees. Clin Orthop Relat Res. 1985; (198):106-9.

5. Lane NE, Michel B, Bjorkengren A, et al. The risk of osteoarthritis with running and aging: a 5-year longitudinal study. J Rheumatol. 1993;20(3):461-8.

6. Cheng Y, Macera CA, Davis DR, et al. Physical activity and selfreported, physician-diagnosed osteoarthritis: is physical activity a risk factor? J Clin Epidemiol. 2000;53(3):315-22.

7. Cymet TC, Sinkov V. Does long-distance running cause osteoarthritis? J Am Osteopath Assoc. 2006;106(6):342-5.

8. Stahl R, Luke A, Ma CB, et al. Prevalence of pathologic findings in asymptomatic knees of marathon runners before and after a competition in comparison with physically active subjects - a 3.0 T magnetic resonance imaging study. Skeletal Radiol. 2008;37 (7):627-38.

9. Krampla W, Mayrhofer R, Malcher J, et al. MR imaging of the knee in marathon runners before and after competition. Skeletal Radiol. 2001;30(2):72-6.

10. Hohmann E, Wortler K, Imhoff AB. MR imaging of the hip and knee before and after marathon running. Am J Sports Med. 2004;32(1):55-9.

11. Kursunoglu-Brahme S, Schwaighofer B, Gundry C, et al. Jogging causes acute changes in the knee joint: an MR study in normal volunteers. AJR Am J Roentgenol. 1990;154(6):1233-5.

12. Shellock FG, Deutsch AL, Mink JH, et al. Do asymptomatic marathon runners have an increased prevalence of meniscal abnormalities? an MR study of the knee in 23 volunteers. AJR Am J Roentgenol. 1991;157(6):1239-41.

13. Krampla WW, Newrkla SP, Kroener AH, et al. Changes on magnetic resonance tomography in the knee joints of marathon runners: a 10year longitudinal study. Skeletal Radiol. 2008;37(7):619-26.

14. Schueller-Weidekamm C, Schueller G, Uffmann M, et al. Does marathon running cause acute lesions of the knee? Evaluation with magnetic resonance imaging. Eur Radiol. 2006;16(10):2179-85.

15. Bashir A, Gray ML, Boutin RD, et al. Glycosaminoglycan in articular cartilage: in vivo assessment with delayed Gd(DTPA)(2-)enhanced MR imaging. Radiology. 1997;205(2):551-8.

16. Rauscher I, Stahl R, Cheng J, et al. Meniscal measurements of T1rho and T2 at MR imaging in healthy subjects and patients with osteoarthritis. Radiology. 2008;249(2):591-600.

17. Stahl R, Luke A, Li X, et al. T1rho, T2 and focal knee cartilage abnormalities in physically active and sedentary healthy subjects versus early OA patients - a 3.0-Tesla MRI study. Eur Radiol. 2009;19(1):132-43.

18. Mosher TJ, Dardzinski BJ. Cartilage MRI T2 relaxation time mapping: overview and applications. Semin Musculoskelet Radiol. 2004;8(4):355-68.

19. Bolbos RI, Link TM, Ma CB, et al. T1rho relaxation time of the meniscus and its relationship with T1rho of adjacent cartilage in knees with acute ACL injuries at $3 \mathrm{~T}$. Osteoarthritis Cartilage. 2009;17(1):12-8.

20. Regatte RR, Akella SV, Lonner JH, et al. T1rho relaxation mapping in human osteoarthritis (OA) cartilage: comparison of T1rho with T2. J Magn Reson Imaging. 2006;23(4):547-53.

21. Krishnan N, Shetty SK, Williams A, et al. Delayed gadoliniumenhanced magnetic resonance imaging of the meniscus: an index of meniscal tissue degeneration? Arthritis Rheum. 2007;56(5):1507-11.

22. Trattnig S, Domayer S, Welsch GW, et al. MR imaging of cartilage and its repair in the knee-a review. Eur Radiol. 2009;19 (7):1582-94.

23. Li X, Benjamin Ma C, Link TM, et al. In vivo $\mathrm{T}(1$ rho) and $\mathrm{T}(2)$ mapping of articular cartilage in osteoarthritis of the knee using 3 T MRI. Osteoarthritis Cartilage. 2007;15(7):789-97.

24. Li X, Han ET, Busse RF, et al. In vivo $T(1$ rho) mapping in cartilage using 3D magnetization-prepared angle-modulated par- 
titioned k-space spoiled gradient echo snapshots (3D MAPSS). Magn Reson Med. 2008;59(2):298-307.

25. Peterfy CG, Guermazi A, Zaim S, et al. Whole-Organ Magnetic Resonance Imaging Score (WORMS) of the knee in osteoarthritis. Osteoarthritis Cartilage. 2004;12(3):177-90.

26. Recht MP, Piraino DW, Paletta GA, et al. Accuracy of fatsuppressed three-dimensional spoiled gradient-echo FLASH MR imaging in the detection of patellofemoral articular cartilage abnormalities. Radiology. 1996;198(1):209-12.

27. Noyes FR, Stabler CL. A system for grading articular cartilage lesions at arthroscopy. Am J Sports Med. 1989;17(4):505-13.

28. Gluer CC, Blake G, Lu Y, et al. Accurate assessment of precision errors: how to measure the reproducibility of bone densitometry techniques. Osteoporos Int. 1995;5(4):262-70.

29. Mosher TJ, Smith HE, Collins C, et al. Change in knee cartilage T2 at MR imaging after running: a feasibility study. Radiology. 2005;234(1):245-9.

30. Stahl R, Blumenkrantz G, Carballido-Gamio J, et al. MRI-derived T2 relaxation times and cartilage morphometry of the tibiofemoral joint in subjects with and without osteoarthritis during a 1-year follow-up. Osteoarthritis Cartilage. 2007;15(11):1225-34.

31. Liess C, Lusse S, Karger N, et al. Detection of changes in cartilage water content using MRI T2-mapping in vivo. Osteoarthritis Cartilage. 2002;10(12):907-13.

32. Mlynarik V, Trattnig S, Huber M, et al. The role of relaxation times in monitoring proteoglycan depletion in articular cartilage. $\mathrm{J}$ Magn Reson Imaging. 1999;10(4):497-502.
33. Borthakur A, Shapiro EM, Beers J, et al. Sensitivity of MRI to proteoglycan depletion in cartilage: comparison of sodium and proton MRI. Osteoarthritis Cartilage. 2000;8(4):288-93.

34. Toffanin R, Mlynarik V, Russo S, et al. Proteoglycan depletion and magnetic resonance parameters of articular cartilage. Arch Biochem Biophys. 2001;390(2):235-42.

35. Watrin-Pinzano A, Ruaud JP, Olivier P, et al. Effect of proteoglycan depletion on $\mathrm{T} 2$ mapping in rat patellar cartilage. Radiology. 2005;234(1):162-70.

36. Akella SV, Regatte RR, Gougoutas AJ, et al. Proteoglycaninduced changes in T1rho-relaxation of articular cartilage at $4 \mathrm{~T}$. Magn Reson Med. 2001;46(3):419-23.

37. McNicol D, Roughley PJ. Extraction and characterization of proteoglycan from human meniscus. Biochem J. 1980;185 (3):705-13.

38. Duvvuri U, Goldberg AD, Kranz JK, et al. Water magnetic relaxation dispersion in biological systems: the contribution of proton exchange and implications for the noninvasive detection of cartilage degradation. Proc Natl Acad Sci USA. 2001;98(22):12479-84.

39. Regatte RR, Akella SV, Borthakur A, et al. Proteoglycan depletion-induced changes in transverse relaxation maps of cartilage: comparison of T2 and T1rho. Acad Radiol. 2002;9 (12):1388-94.

40. Pap G, Eberhardt R, Sturmer I, et al. Development of osteoarthritis in the knee joints of Wistar rats after strenuous running exercise in a running wheel by intracranial self-stimulation. Pathol Res Pract. 1998;194(1):41-7. 